COMMUNICATIONS IN ALGEBRA ${ }^{\circledR}$

Vol. 31, No. 5, pp. 2207-2217, 2003

\title{
Dimension of the Mesh Algebra of a Finite Auslander-Reiten Quiver
}

\author{
Ragnar-Olaf Buchweitz ${ }^{1, *}$ and Shiping Liu ${ }^{2}$ \\ ${ }^{1}$ Department of Mathematics, University of Toronto, \\ Toronto, Ontario, Canada \\ ${ }^{2}$ Département de Mathématiques, Université de Sherbrooke, \\ Sherbrooke, Québec, Canada
}

\begin{abstract}
Translation quivers appear naturally in the representation theory of finite dimensional algebras; see, for example, Bongartz and Gabriel (Bongartz, K., Gabriel, P., (1982). Covering spaces in representation theory. Invent. Math. 65:331-378.). A translation quiver defines a mesh algebra over any field. A natural question arises as to whether or not the dimension of the mesh algebra depends on the field. The purpose of this note is to show that the dimension of the mesh algebra of a finite Auslander-Reiten quiver over a field is a purely combinatorial invariant of this quiver. Indeed, our proof yields a combinatorial algorithm for computing this dimension. As a further
\end{abstract}

*Correspondence: Ragnar-Olaf Buchweitz, Department of Mathematics, University of Toronto, Toronto, Ont., Canada M5S 3G3; E-mail: ragnar@math. toronto.edu. 
application, one may use then semicontinuity of Hochschild cohomology of algebras as in Buchweitz and Liu (Buchweitz, R.-O., Lui, S. Hochschild cohomology and representation-finite algebras. Preprint.) to conclude that a finite Auslander-Reiten quiver contains no oriented cycle if its mesh algebra over some field admits no outer derivation.

Key Words: Translation quiver; Mesh algebra; Auslander-Reiten quiver.

\section{SIMPLY CONNECTED TRANSLATION QUIVERS}

Throughout this note, $\Gamma$ denotes a translation quiver with translation $\tau$; see Bongartz and Gabriel (1982, (1.1)). We assume that $\Gamma$ contains neither loops nor multiple arrows but that each non-projective vertex is the end-point of at least one arrow. One defines the orbit graph $\mathcal{O}(\Gamma)$ of $\Gamma$ as follows: the $\tau$-orbit of a vertex $x$ is the set $o(x)$ of vertices of the form $\tau^{n}(x)$ with $n \in \mathbb{Z}$; the vertices of $\mathcal{O}(\Gamma)$ are the $\tau$-orbits of $\Gamma$, and there exists an edge $o(x)-o(y)$ in $\mathcal{O}(\Gamma)$ if $\Gamma$ contains an arrow $a \rightarrow b$ or $b \rightarrow a$ with $a \in o(x)$ and $b \in o(y)$. If $\Gamma$ contains no oriented cycle, then $\mathcal{O}(\Gamma)$ is the graph $G_{\Gamma}$ defined in Bongartz and Gabriel (1982, (4.2)). Now $\Gamma$ is called simply connected if $\Gamma$ contains no oriented cycle and $\mathcal{O}(\Gamma)$ is a tree. By Bongartz and Gabriel (1982, (1.6), (4.1), (4.2)), this definition is equivalent to that given in Bongartz and Gabriel $(1982,(1.6))$.

Let $p: x_{0} \rightarrow x_{1} \rightarrow \cdots \rightarrow x_{r}$ be a path in $\Gamma$. Then $p$ induces a walk $w(p): o\left(x_{0}\right)-o\left(x_{1}\right)-\cdots-o\left(x_{r}\right)$ in $\mathcal{O}(\Gamma)$, and $w(p)$ in turn determines a unique reduced walk $w_{\operatorname{red}(p)}$. Recall that the path $p$ is called sectional if $x_{i-1} \neq \tau x_{i+1}$ for each $0<i<r$. Moreover, for a vertex $x \in \Gamma$, we denote by $x^{+}$and by $x^{-}$the set of immediate successors and that of immediate predecessors of $x$, respectively.

We recall that a sequence $a_{0} \frac{e_{1}}{{ }_{1}} a_{1}-\cdots-a_{r-1} \frac{e_{r}}{a_{r}} a_{r}$ of edges of a graph such that $e_{i} \neq e_{i+1}$ for all $1 \leq i<r$ is called a reduced walk. A vertex of a graph is considered as a trivial reduced walk.

Lemma 1.1. Assume that $\Gamma$ contains no oriented cycle.

(1) Let $x$ be a vertex of $\Gamma$. If $x_{1}, x_{2} \in x^{-}$or $x_{1}, x_{2} \in x^{+}$, then $o\left(x_{1}\right)=o\left(x_{2}\right)$ if and only if $x_{1}=x_{2}$.

(2) If $p$ is a sectional path in $\Gamma$, then $w(p)$ is a reduced walk in $\mathcal{O}(\Gamma)$.

Proof. (1) It suffices to consider the case where $x_{1}, x_{2} \in x^{-}$. Suppose that $o\left(x_{1}\right)=o\left(x_{2}\right)$. We may assume that $x_{2}=\tau^{r} x_{1}$ for some $r \geq 0$. 
If $r>0$, then $x \rightarrow \tau^{r-1} x_{1} \rightarrow \cdots \rightarrow x_{1} \rightarrow x$ would be an oriented cycle. Thus $r=0$, that is $x_{1}=x_{2}$.

(2) Let $p: x_{0} \rightarrow x_{1} \rightarrow \cdots \rightarrow x_{r}$ be a sectional path in $\Gamma$. If $w(p)$ is not reduced, then $o\left(x_{i-1}\right)=o\left(x_{i+1}\right)$ for some $0<i<r$. However, this means that $x_{i-1}=\tau^{m} x_{i+1}$ for some integer $m$. As $\Gamma$ contains no oriented cycle, $m$ is necessarily positive. In particular, $\tau x_{i+1}$ exists, and then by part (1), $x_{i-1}=\tau x_{i+1}$ since $x_{i-1}, \tau x_{i+1} \in x_{i}^{-}$. This contradicts the hypothesis that $p$ is sectional. The proof of the lemma is completed.

Lemma 1.2. If $\Gamma$ is simply connected, then a sectional path in $\Gamma$ is the only path between its endpoints.

Proof. Assume that $\Gamma$ is simply connected. We shall prove the lemma by induction on the length of a sectional path. The lemma is trivially true if the length is zero, as $\Gamma$ contains no oriented cycle. Assume that $r>0$ and the lemma holds for sectional paths of length less than $r$. Let

$$
p: x=y_{0} \rightarrow y_{1} \rightarrow \cdots \rightarrow y_{r-1} \rightarrow y_{r}=y
$$

be a sectional path. Then the path $u$ obtained from $p$ by dropping the initial arrow is the unique path from $y_{1}$ to $y$. Note that $u$ does not start with $y_{1} \rightarrow \tau^{-} x$ as $p$ is sectional. Now let

$$
q: x=x_{0} \rightarrow x_{1} \rightarrow \cdots \rightarrow x_{s-1} \rightarrow x_{s}=y
$$

be another path in $\Gamma$ from $x$ to $y$. We claim first that $o(x) \neq o\left(x_{t}\right)$ for $0<t \leq s$. Otherwise, $x_{t}=\tau^{-m} x$ for some such $t$ and some integer $m$. Using again the fact that $\Gamma$ contains no oriented cycle, we have $m>0$ and consequently a path from $\tau^{-} x$ to $\tau^{-m} x$. Further the initial arrow $x \rightarrow y_{1}$ gives rise to an arrow $y_{1} \rightarrow \tau^{-} x$. Thus we obtain a path

$$
y_{1} \rightarrow \tau^{-} x \rightarrow \cdots \rightarrow \tau^{-m} x=x_{t} \rightarrow x_{t+1} \rightarrow \cdots \rightarrow x_{s-1} \rightarrow y,
$$

which is different from $u$, a contradiction.

Thus, $o(x) \neq o\left(x_{t}\right)$ for $0<t \leq s$, as claimed. It follows that $o(x)-$ $o\left(x_{1}\right)$ is the initial edge of the reduced walk $w_{\text {red }(q)}$ from $o(x)$ to $o(y)$ in $\mathcal{O}(\Gamma)$. Now $w(p)$ is a reduced walk from $o(x)$ to $o(y)$ whose initial edge is $o(x)-o\left(y_{1}\right)$. As $\mathcal{O}(\Gamma)$ is a tree, the edge $o(x)-o\left(x_{1}\right)$ coincides with the edge $o(x)-o\left(y_{1}\right)$. In particular, $o\left(x_{1}\right)=o\left(y_{1}\right)$. Thus $x_{1}=y_{1}$, by Lemma 1.1(1). Since $\Gamma$ contains no multiple arrow, the initial arrow $x \rightarrow x_{1}$ of $q$ is the same as that of $p$. Hence $p=q$ by the inductive hypothesis. This completes the proof of the lemma.

For a vertex $x$ of $\Gamma$, we define the forward cone $\Gamma_{x}$ of $x$ to be the set of all successors of $x$ in $\Gamma$. 
Lemma 1.3. Assume that $\Gamma$ is simply connected. Let $x$ be a vertex of $\Gamma$ and assume that

$$
p: x_{0} \rightarrow x_{1} \rightarrow \cdots \rightarrow x_{m-1} \rightarrow x_{m}
$$

is a non-trivial sectional path in $\Gamma$ with $x_{0} \in \Gamma_{x}$ and $x_{1}$ projective. For each $1 \leq i \leq m$, the vertex $x_{i-1}$ is then the only immediate predecessor of $x_{i}$ that belongs to $\Gamma_{x}$.

Proof. As $x_{0} \in \Gamma_{x}$, there exists a path

$$
q: x=y_{0} \rightarrow y_{1} \rightarrow \cdots \rightarrow y_{r-1} \rightarrow y_{r}=x_{0} \rightarrow x_{1}
$$

in $\Gamma$. Since $x_{1}$ is projective and $\Gamma$ contains no oriented cycle, $o\left(y_{i}\right) \neq o\left(x_{1}\right)$ for all $0 \leq i \leq r$. Therefore, the associated reduced walk $w_{\operatorname{red}(q)}$ from $o(x)$ to $o\left(x_{1}\right)$ in $\mathcal{O}(\Gamma)$ ends with the edge $o\left(x_{0}\right)-o\left(x_{1}\right)$. If

$$
u: x=a_{0} \rightarrow a_{1} \rightarrow \cdots \rightarrow a_{s-1} \rightarrow a_{s}=x_{1}
$$

is a second such path, by the same argument, the reduced walk $w_{\text {red }}(u)$ from $o(x)$ to $o\left(x_{1}\right)$ ends with the edge $o\left(a_{s-1}\right)-o\left(x_{1}\right)$. This reduced walk coincides with the reduced walk $w_{\text {red }}(q)$, as $\mathcal{O}(\Gamma)$ is a tree. Consequently, $o\left(a_{s-1}\right)=o\left(x_{0}\right)$, and then, by Lemma 1.1(1), $a_{s-1}=x_{0}$. This shows that the lemma holds for $m=1$. Assume now that $m>1$ and that the lemma holds for $m-1$. By Lemma 1.1(2),

$$
w(p): o\left(x_{0}\right)-o\left(x_{1}\right)-\cdots-o\left(x_{m-1}\right)-o\left(x_{m}\right)
$$

is a reduced walk in $\mathcal{O}(\Gamma)$. Combining $w(p)$ with the reduced walk $w_{\text {red }}(q)$ results in a reduced walk

$$
w: o(x)-\cdots-o\left(x_{0}\right)-o\left(x_{1}\right)-\cdots-o\left(x_{m-1}\right)-o\left(x_{m}\right) .
$$

Let $v: x=z_{0} \rightarrow z_{1} \rightarrow \cdots \rightarrow z_{t-1} \rightarrow z_{t}=x_{m}$ be a path in $\Gamma$. If $o\left(x_{m}\right)=$ $o\left(z_{i}\right)$ for some $0 \leq i<t$, then $z_{i}=\tau^{n} x_{m}$. Now $n>0$ as $\Gamma$ contains no oriented cycle. This implies that $\tau x_{m}$ is in $\Gamma_{x}$, and then $\tau x_{m}$ and $x_{m-2}$ were different immediate predecessors of $x_{m-1}$ in $\Gamma_{x}$, contrary to the inductive hypothesis. Thus, $o\left(x_{m}\right) \neq o\left(z_{i}\right)$ for each $0 \leq i<t$, and so $u$ induces a reduced walk from $o(x)$ to $o\left(x_{m}\right)$ that ends with the edge $o\left(z_{t-1}\right)$ $o\left(x_{m}\right)$. Again $o\left(z_{s-1}\right)=o\left(x_{m-1}\right)$ since $\mathcal{O}(\Gamma)$ is a tree, and hence $z_{s-1}=x_{m-1}$, by Lemma 1.1. This completes the induction, thus, the proof of the lemma.

Lemma 1.4. Assume that $\Gamma$ is simply connected. If $x, y$ are vertices of $\Gamma$, then all paths from $x$ to $y$ in $\Gamma$ are of the same length. 
Proof. We fix a vertex $x \in \Gamma$. Let $x=x_{0} \rightarrow x_{1} \rightarrow \cdots \rightarrow x_{r}=y$ be a path in $\Gamma$. We shall use induction on $i$ to prove that all paths from $x$ to $x_{i}$ are of length $i$. This is trivially true for $i=0$ since $\Gamma$ contains no oriented cycle. Assume that $1 \leq i \leq r$ and all paths from $x$ to $x_{i-1}$ are of length $i-1$. Assume further that $q: x=y_{0} \rightarrow y_{1} \rightarrow \cdots \rightarrow y_{s}=x_{i}$ is another path. Note that $s>0$.

Let $t$ with $0 \leq t<s$ be the smallest integer such that the path $y_{t} \rightarrow \cdots \rightarrow y_{s}$ is sectional. If $t=0$, then $s=i$ by Lemma 1.2. Assume that $t>0$. Then $y_{t-1}=\tau y_{t+1}$ by the minimality of $t$. If some $y_{j}$ with $t+1<$ $j \leq s$ is projective, then $y_{s-1}=x_{i-1}$ by Lemma 1.3. Therefore, $s-1=i-1$ by the inductive hypothesis. Otherwise, $\Gamma$ contains a path

$$
x \rightarrow y_{1} \rightarrow \cdots \rightarrow y_{t-1}=\tau y_{t+1} \rightarrow \cdots \rightarrow \tau y_{s} \rightarrow x_{i-1} .
$$

By the inductive hypothesis, $i-1=(t-1)+(s-t)$, whence $s=i$. This completes the induction, and hence the proof of the lemma.

\section{MESH ALGEBRAS}

The objective of this section is to prove our main result. For this purpose, we need to recall some basic notions and results on linear categories from Bongartz and Gabriel (1982). Throughout this section, $k$ denotes an algebraically closed field and $\Lambda$ a locally bounded $k$-category. There exists a unique locally finite quiver $Q_{\Lambda}$, called the ordinary quiver of $\Lambda$, such that $\Lambda \cong k Q_{\Lambda} / I_{\Lambda}$, where $k Q_{\Lambda}$ is the path category of $Q_{\Lambda}$ over $k$ and $I_{A}$ is an ideal contained in the ideal of $k Q_{\Lambda}$ generated by the paths of length two. The radical $\mathfrak{R} \Lambda$ of $\Lambda$ is the ideal generated by the non-invertible morphisms. Set $\mathfrak{R}^{0} \Lambda=\Lambda$ and define $\mathfrak{R}^{n} \Lambda$ for $n \geq 1$ to be the ideal generated by the composites of $n$ morphisms from $\mathfrak{R} \Lambda$. Denote by $\bmod \Lambda$ the category of finite dimensional right $\Lambda$-modules and by ind $\Lambda$ the full subcategory of $\bmod \Lambda$ generated by a chosen complete set of representatives of isomorphim classes of indecomposable modules in $\bmod \Lambda$.

We shall denote by $k(\Gamma)$ the mesh category of $\Gamma$ over $k$; see Bongartz and Gabriel $(1982,(2.5))$. Note that for each $n \geq 0$, the ideal $\mathfrak{R}^{n} k(\Gamma)$ is generated by the classes of paths of length $n$ in $\Gamma$ modulo the mesh ideal. If $\Gamma$ is finite, then $\bigoplus_{x, y \in \Gamma} k(\Gamma)(x, y)$ is an $k$-algebra, called the mesh algebra of $\Gamma$ over $k$ and denoted again by $k(\Gamma)$. Note that we can define in the same way the mesh algebra $R(\Gamma)$ of $\Gamma$ over any commutative ring $R$.

Assume now that $\Lambda$ is locally representation-finite, that is, every object $x$ of $\Lambda$ lies in the support of only finitely many modules in ind $\Lambda$, or equivalently, ind $\Lambda$ is locally bounded. The Auslander-Reiten quiver 
$\Gamma_{\Lambda}$ of $\Lambda$ is a translation quiver with respect to the Auslander-Reiten translation $\tau_{\Lambda}=D T r$, the dual of the transpose. Recall that $\Lambda$ is called standard, (Bongartz and Gabriel, 1982, (5.1)), if ind $\Lambda \cong k\left(\Gamma_{\Lambda}\right)$, and simply connected, (Bongartz and Gabriel, 1982, (6.1)), if $\Gamma_{\Lambda}$ is a simply connected translation quiver. It follows from (Bretscher and Gabriel, 1983, (3.1)) that standardness in this sense is equivalent to that defined in (Bautista et al., 1985, (1.11)). If $\Gamma_{\Lambda}$ contains no oriented cycle, then the ordinary quiver $Q_{\Lambda}$ of $\Lambda$ contains no loop as ind $\Lambda$ is locally bounded, and consequently, $\Lambda$ is standard (Bautista et al., 1985, (9.6)). In particular, $\Lambda$ is standard if it is simply connected.

We say that $\Gamma$ is an Auslander-Reiten quiver over $k$ if $\Gamma \cong \Gamma_{\Lambda}$ for some locally representation-finite $k$-category $\Lambda$. In this case, $k(\Gamma)$ is a locally bounded $k$-category. It follows from Brenner's result (Brenner, 1986, (5.1)) that if $\Gamma$ is an Auslander-Reiten quiver over $k$, then it is an Auslander-Reiten quiver over any algebraically closed field. In the sequel, we shall simply say that $\Gamma$ is an Auslander-Reiten quiver with no reference to a ground field.

Lemma 2.1. Let $\Gamma$ be a simply connected Auslander-Reiten quiver, and let $k$, $l$ be algebraically closed fields. For any pair $x, y$ of vertices of $\Gamma$ and any integer $n \geq 0$, one has then

$$
\operatorname{dim}_{k} \Re^{n} k(\Gamma)(x, y)=\operatorname{dim}_{l} \mathfrak{R}^{n} l(\Gamma)(x, y) .
$$

Proof. Let $\Lambda$ and $\Sigma$ be a locally representation-finite $k$-category and l-category, respectively, such that $\Gamma \cong \Gamma_{\Lambda} \cong \Gamma_{\Sigma}$. Then $\Lambda$ and $\Sigma$ are simply connected, in particular, $\Lambda$ and $\Sigma$ are standard. Thus $k(\Gamma) \cong k\left(\Gamma_{\Lambda}\right) \cong$ ind $\Lambda$, and similarly $l(\Gamma) \cong$ ind $\Sigma$.

We fix a vertex $x$ of $\Gamma$. If $y \notin \Gamma_{x}$, then $\operatorname{dim}_{k} k(\Gamma)(x, y)=0=$ $\operatorname{dim}_{l} l(\Gamma)(x, y)$. Assume now that $y \in \Gamma_{x}$. By Lemma 1.4, all paths from $x$ to $y$ have the same length, which we denote by $d(x, y)$.

If $d(x, y)=0$, then $\operatorname{dim}_{k} k(\Gamma)(x, y)=1=\operatorname{dim}_{l} l(\Gamma)(x, y)$. Now assume that $d(x, y)=r>0$ and $\operatorname{dim}_{k} k(\Gamma)(x, z)=\operatorname{dim}_{l} l(\Gamma)(x, z)$ for all $z \in \Gamma_{x}$ with $d(x, z)<r$. Assume that $y^{-}=\left\{x_{1}, \ldots, x_{s}\right\}$. For each $1 \leq i \leq s$, either $x_{i} \notin \Gamma_{x}$, or $x_{i} \in \Gamma_{x}$ and then $d\left(x, x_{i}\right)=r-1$. It follows thus from the inductive hypothesis that $\operatorname{dim}_{k} k(\Gamma)\left(x, x_{i}\right)=\operatorname{dim}_{l} l(\Gamma)\left(x, x_{i}\right)$ for all $1 \leq i \leq s$.

Let $\phi: \Gamma \rightarrow \Gamma_{\Lambda}$ be an isomorphism that exists by our assumption. Set $M=\phi(x), N=\phi(y)$, and $M_{i}=\phi\left(x_{i}\right)$ for $1 \leq i \leq s$. If $y$ is not a projective vertex, then $N$ is not a projective module. There exists thus an almost split sequence

$$
0 \rightarrow \tau_{\Lambda} N \rightarrow M_{1} \oplus \cdots \oplus M_{s} \rightarrow N \rightarrow 0
$$


in $\bmod \Lambda$. Note that $M \nsubseteq N$, since $\Gamma_{\Lambda}$ contains no oriented cycle and $r>0$. Accordingly, applying $\operatorname{Hom}_{\Lambda}(M,-)$ to the above almost split sequence yields an exact sequence

$$
0 \rightarrow \operatorname{Hom}_{\Lambda}\left(M, \tau_{\Lambda} N\right) \rightarrow \bigoplus_{i=1}^{s} \operatorname{Hom}_{\Lambda}\left(M, M_{i}\right) \rightarrow \operatorname{Hom}_{\Lambda}(M, N) \rightarrow 0,
$$

and counting dimensions gives rise to the following equalities:

$$
\begin{aligned}
\operatorname{dim}_{k} k(\Gamma)(x, y) & =\operatorname{dim}_{k} \operatorname{Hom}_{\Lambda}(M, N) \\
& =\sum_{i=1}^{s} \operatorname{dim}_{k} \operatorname{Hom}_{\Lambda}\left(M, M_{i}\right)-\operatorname{dim}_{k} \operatorname{Hom}_{\Lambda}\left(M, \tau_{\Lambda} N\right) \\
& =\sum_{i=1}^{s} \operatorname{dim}_{k} k(\Gamma)\left(x, x_{i}\right)-\operatorname{dim}_{k} k(\Gamma)(x, \tau y) .
\end{aligned}
$$

If $y$ is a projective vertex, then $N$ is a projective module, and so

$$
\begin{aligned}
\operatorname{dim}_{k} k(\Gamma)(x, y) & =\operatorname{dim}_{k} \operatorname{Hom}_{\Lambda}(M, N) \\
& =\sum_{i=1}^{s} \operatorname{dim}_{k} \operatorname{Hom}_{\Lambda}\left(M, M_{i}\right) \\
& =\sum_{i=1}^{s} \operatorname{dim}_{k} k(\Gamma)\left(x, x_{i}\right) .
\end{aligned}
$$

Similarly, $\operatorname{dim}_{l} l(\Gamma)(x, y)=\sum_{i=1}^{s} \operatorname{dim}_{l} l(\Gamma)\left(x, x_{i}\right)-\operatorname{dim}_{l} l(\Gamma)(x, \tau y)$, if $y$ is non-projective; and otherwise, $\operatorname{dim}_{l} l(\Gamma)(x, y)=\sum_{i=1}^{s} \operatorname{dim}_{l} l(\Gamma)\left(x, x_{i}\right)$. In case $y$ is non-projective, either $\tau y \notin \Gamma_{x}$ or $\tau y \in \Gamma_{x}$ with $d(x, \tau y)=r-2$. Thus $\operatorname{dim}_{k} k(\Gamma)(x, \tau y)=\operatorname{dim}_{l} l(\Gamma)(x, \tau y)$ by the inductive hypothesis. Therefore, $\operatorname{dim}_{k} k(\Gamma)(x, y)=\operatorname{dim}_{l} l(\Gamma)(x, y)$ in either case.

Finally, let us fix an integer $n \geq 1$. If $\Gamma$ contains no path from $x$ to $y$ of length $\geq n$, then $\mathfrak{R}^{n} k(\Gamma)(x, y)=0=\mathfrak{R}^{n} l(\Gamma)(x, y)$. Otherwise, by Lemma 1.4, all paths from $x$ to $y$ are of length $\geq n$. Hence $\mathfrak{R}^{n} k(\Gamma)(x, y)=k(\Gamma)(x, y)$ and $\mathfrak{R}^{n} l(\Gamma)(x, y)=l(\Gamma)(x, y)$. Thus $\operatorname{dim}_{k} \mathfrak{R}^{n} k(\Gamma)(x, y)=\operatorname{dim}_{l} \mathfrak{R}^{n} l(\Gamma)(x, y)$. This completes the proof of the lemma.

Note that if $\Gamma$ is a finite Auslander-Reiten quiver, then $k(\Gamma)$ is finite dimensional over $k$. In order to show that the mesh algebra $F(\Gamma)$ of $\Gamma$ over any other field $F$ is of dimension $\operatorname{dim}_{k} k(\Gamma)$, recall that a $k$-linear functor $\pi: \tilde{\Lambda} \rightarrow \Lambda$ of locally bounded $k$-categories is a covering functor, Bongartz and Gabriel (1982, (3.1)), if for all objects $x, y$ of $\Lambda$, the fibre $\pi^{-}(x)$ is non-empty, and for each $\tilde{x} \in \pi^{-}(x)$, the map $\pi$ induces 
$k$-isomorphisms

$$
\bigoplus_{\tilde{y} \in \pi^{-}(y)} \tilde{\Lambda}(\tilde{x}, \tilde{y}) \stackrel{\cong}{\longrightarrow} \Lambda(x, y) \text { and } \bigoplus_{\tilde{y} \in \pi^{-}(y)} \tilde{\Lambda}(\tilde{y}, \tilde{x}) \stackrel{\cong}{\longrightarrow} \Lambda(y, x) .
$$

It is easy to see that $\pi$ induces, for each $n \geq 1$, a $k$-isomorphism

$$
\bigoplus_{\tilde{y} \in \pi^{-}(y)} \mathfrak{R}^{n} \tilde{\Lambda}(\tilde{x}, \tilde{y}) \stackrel{\cong}{\longrightarrow} \mathfrak{R}^{n} \Lambda(x, y) .
$$

Theorem 2.2. Let $\Gamma$ be a finite Auslander-Reiten quiver. For each $n \geq 0$, there exists $d_{n} \geq 0$ such that $\operatorname{dim}_{F} \Re^{n} F(\Gamma)=d_{n}$ for any field $F$.

Proof. We fix an algebraically closed field $k$, and let $F$ be an arbitrary field with algebraic closure $l$. Clearly, $\mathfrak{R}^{n} l(\Gamma)=\mathfrak{R}^{n} F(\Gamma) \otimes_{F} l$ for each $n \geq 0$, whence we need only to show that $\operatorname{dim}_{k} \Re^{n} k(\Gamma)=\operatorname{dim}_{l} \Re^{n} l(\Gamma)$ for each $n \geq 0$. Furthermore, it suffices to show that $\operatorname{dim}_{k} \mathfrak{R}^{n} k(\Gamma)(x, y)=$ $\operatorname{dim}_{l} \mathfrak{R}^{n} l(\Gamma)(x, y)$ for each pair $x, y$ of vertices in $\Gamma$ and each $n \geq 0$. Let $\pi: \tilde{\Gamma} \rightarrow \Gamma$ be the universal covering of $\Gamma$; see Bongartz and Gabriel (1982, (1.3)). Then $\pi$ induces covering functors $k(\tilde{\Gamma}) \rightarrow k(\Gamma)$ and $l(\tilde{\Gamma}) \rightarrow l(\Gamma)$; see Bongartz and Gabriel (1982, (3.1)).

Let now $\Lambda$ be a representation-finite $k$-category such that $\Gamma \cong \Gamma_{\Lambda}$. Let $\tilde{\Lambda}$ be the full subcategory of $k(\tilde{\Gamma})$ generated by the projective vertices. According to Bongartz and Gabriel (1982, (2.4)), $\tilde{\Lambda}$ is a locally representation-finite $k$-category such that $\Gamma_{\tilde{\Lambda}} \cong \tilde{\Gamma}$. We fix a vertex $\tilde{x} \in \pi^{-}(x)$ and an integer $n \geq 0$. then

$$
\mathfrak{R}^{n} k(\Gamma)(x, y) \cong \bigoplus_{\tilde{y} \in \pi^{-}(y)} \mathfrak{R}^{n} k(\tilde{\Gamma})(\tilde{x}, \tilde{y}) .
$$

Therefore, $\operatorname{dim}_{k} \mathfrak{R}^{n} k(\Gamma)(x, y)=\sum_{\tilde{y} \in \pi^{-}(y)} \operatorname{dim}_{k} \mathfrak{R}^{n} k(\tilde{\Gamma})(\tilde{x}, \tilde{y})$. Similarly, one can show that

$$
\operatorname{dim}_{l} \mathfrak{R}^{n} l(\Gamma)(x, y)=\sum_{\tilde{y} \in \pi^{-}(y)} \operatorname{dim}_{l} \mathfrak{R}^{n} l(\tilde{\Gamma})(\tilde{x}, \tilde{y}) .
$$

Now by Lemma $2.1, \operatorname{dim}_{k} \Re^{n} k(\tilde{\Gamma})(\tilde{x}, \tilde{y})=\operatorname{dim}_{l} \Re^{n} l(\tilde{\Gamma})(\tilde{x}, \tilde{y})$, as $\tilde{\Gamma}$ is simply connected. This completes the proof of the theorem.

Remark. It seems plausible that the above result should hold true for any translation quiver $\Gamma$ with $k(\Gamma)$ being finite dimensional. However, 
the analogue to the exact sequence $(*)$ in the proof of Lemma 2.1 fails for a general translation quiver.

We shall now show that the mesh algebra of a finite AuslanderReiten quiver over a commutative ring is free of finite rank. For this purpose, we need the following basic result, in which all tensor products are taken over $\mathbb{Z}$.

Lemma 2.3. Let $A$ be a $\mathbb{Z}$-algebra, and let I be a finitely generated ideal such that $A / I$ is finitely generated as $\mathbb{Z}$-module. Assume that there exists a constant $d$ such that $\operatorname{dim}_{F}(A \otimes F)=\operatorname{dim}_{F}(A / I \otimes F)=d$ for any field $F$. Then $I=0$ and $A$ is a free $\mathbb{Z}$-module of rank $d$.

Proof. If $M$ is a finitely generated $\mathbb{Z}$-module such that $\operatorname{dim}_{F}(M \otimes F)=d$ for all fields $F$, then it follows from the structure theorem for finitely generated modules over a principal ideal domain that $M$ is a free $\mathbb{Z}$-module of rank $d$. In particular, $A / I$ is a free $\mathbb{Z}$-module of rank $d$ by our assumption, and it suffices to show that $I=0$.

As $\mathbb{Q}$ is $\mathbb{Z}$-flat, we have an exact sequence $0 \rightarrow I \otimes \mathbb{Q} \rightarrow A \otimes \mathbb{Q} \rightarrow$ $A / I \otimes \mathbb{Q} \rightarrow 0$. Since $A \otimes \mathbb{Q}$ and $A / I \otimes \mathbb{Q}$ are of the same finite dimension over $\mathbb{Q}$ by assumption, we have $I \otimes \mathbb{Q}=0$. This implies that $I$ is a torsion group. Since $I$ is finitely generated as ideal, there exists an integer $m>0$ such that $m I=0$.

We now fix a prime $p$, and let $\mathbb{Z}_{(p)}$ be the localization of $\mathbb{Z}$ at $p \mathbb{Z}$ and $\mathbb{Z}_{p}$ its residue field. Then $\mathbb{Z}_{(p)}$ is flat over $\mathbb{Z}$ and there exists an exact sequence

$$
0 \rightarrow \mathbb{Z}_{(p)} \stackrel{p .}{\rightarrow} \mathbb{Z}_{(p)} \rightarrow \mathbb{Z}_{p} \rightarrow 0,
$$

where $p$ denotes multiplication. Thus we obtain the following exact commutative diagram:

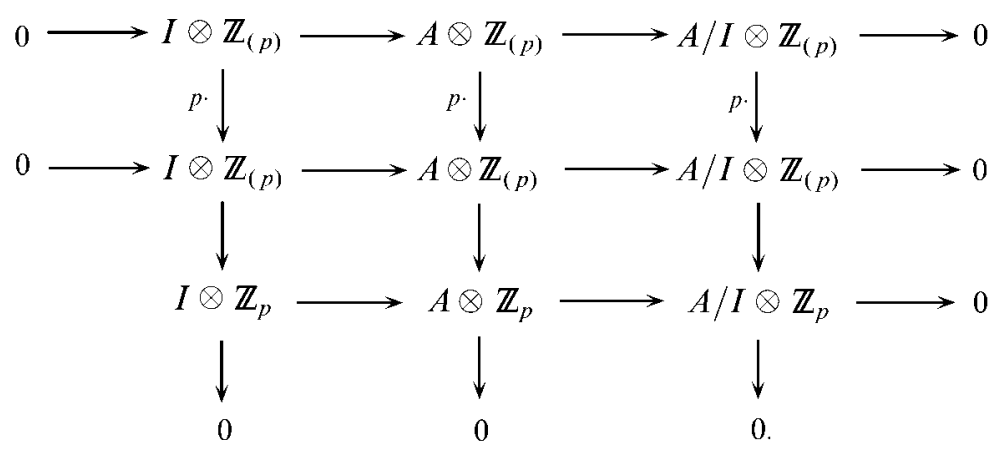


As $A / I$ is free over $\mathbb{Z}$, the $\mathbb{Z}_{(p)}$-module $A / I \otimes \mathbb{Z}_{(p)}$ is free as well. Hence $p \cdot: A / I \otimes \mathbb{Z}_{(p)} \rightarrow A / I \otimes \mathbb{Z}_{(p)}$ is a monomomorphism. So the Snake Lemma yields an exact sequence

$$
0 \rightarrow I \otimes \mathbb{Z}_{p} \rightarrow A \otimes \mathbb{Z}_{p} \rightarrow A / I \otimes \mathbb{Z}_{p} \rightarrow 0 .
$$

Since $A \otimes \mathbb{Z}_{p}$ and $A / I \otimes \mathbb{Z}_{p}$ are of the same finite dimension over $\mathbb{Z}_{p}$, we have $I \otimes \mathbb{Z}_{p}=0$. On the other hand, we have $I \otimes \mathbb{Z}_{p} \cong I / p I$. Hence $p I=I$, for any prime $p$. Therefore, $I=m I=0$, and the proof of the lemma is complete.

Remark. The above lemma fails for the trivial extension $\mathbb{Z} \ltimes(\mathbb{Q} / \mathbb{Z})$, whence finite generation of the ideal $I$ is necessary.

Proposition 2.4. Let $R$ be a commutative ring and $\Gamma$ a finite AuslanderReiten quiver. Then the mesh algebra $R(\Gamma)$ is a free $R$-module of finite rank and that rank is independent of $R$.

Proof. In this proof, all tensor products are taken over $\mathbb{Z}$. Note first that $R(\Gamma) \cong \mathbb{Z}(\Gamma) \otimes R$. It is thus sufficient to show that $\mathbb{Z}(\Gamma)$ is free over $\mathbb{Z}$ of finite rank.

We first fix an algebraically closed field $k$. Since $k(\Gamma)$ is finite dimensional over $k$, there exists $r>0$ such that $\mathfrak{R}^{r} k(\Gamma)=0$. Set $A=\mathbb{Z}(\Gamma)$ and $I=\mathfrak{R}^{r} \mathbb{Z}(\Gamma)$. Then $I$ is finitely generated as an ideal and $A / I$ is finitely generated as $\mathbb{Z}$-module. Let $F$ be an arbitrary field. Under the isomorphism $A \otimes F \cong F(\Gamma)$, the image of $I \otimes F$ in $A \otimes F$ maps onto $\mathfrak{R}^{r} F(\Gamma)$. Hence, $A / I \otimes F \cong F(\Gamma) / \Re^{r} F(\Gamma)$. On the other hand, by Theorem 2.2, $\operatorname{dim}_{F} \mathfrak{R}^{n} F(\Gamma)=\operatorname{dim}_{k} \mathfrak{R}^{n} k(\Gamma)$ for all $n \geq 0$. In particular, $\mathfrak{R}^{r} F(\Gamma)=0$. This yields

$$
\operatorname{dim}_{F}(A / I \otimes F)=\operatorname{dim}_{F} F(\Gamma)=\operatorname{dim}_{F}(A \otimes F),
$$

which is further equal to $\operatorname{dim}_{k} k(\Gamma)$. Now Lemma 2.3 applies and shows that $\mathbb{Z}(\Gamma)=A$ is a free $\mathbb{Z}$-module of finite rank. This completes the proof.

\section{ACKNOWLEDGMENT}

Both authors gratefully acknowledge partial support from the Natural Sciences and Engineering Research Council of Canada. 


\section{REFERENCES}

Auslander, M., Reiten, I., Smalø, S. O. (1995). Representation Theory of Artin Algebras. Cambridge Studies in Advanced Mathematics 36. Cambridge: Cambridge University Press.

Bautista, R., Gabriel, P., Roiter, A. V., Salmerón, L. (1985). Representation-finite algebras and multiplicative basis. Invent. Math. 81: 217-285.

Bongartz, K., Gabriel, P. (1982). Covering spaces in representation theory. Invent. Math. 65:331-378.

Brenner, S. (1986). A Combinatorial Characterisation of Finite AuslanderReiten Quivers. Lecture Notes in Mathematics. Vol. 1177. Berlin: Springer-Verlag, pp. 13-49.

Bretscher, O., Gabriel, P. (1983). The standard form of a representationfinite algebra. Bull. Soc. Math. France 111:21-40.

Buchweitz, R.-O., Liu, S. Hochschild cohomology and representationfinite algebras. Preprint.

Received January 15, 2002

Revised June 18, 2002 
